\title{
An emerging molecular mechanism for the neural vs mesodermal cell fate decision
}

\author{
Roman $\mathrm{A} \mathrm{Li}^{1}$, Kate G Storey ${ }^{1}$ \\ ${ }^{I}$ Division of Cell and Developmental Biology, College of Life Sciences, University of Dundee, UK \\ Cell Research (2011) 21:708-710. doi:10.1038/cr.2011.54; published online 29 March 2011
}

Understanding how primary cell fates are established and maintained in the vertebrate embryo provides important insights that inform directed in vitro differentiation of embryonic stem cells or adult cells that have undergone induced pluripotency. Neural differentiation is of particular interest as new neural cells may contribute to therapeutic approaches to nervous system injury and diseases and provide in vitro disease models for small molecule screening and for determining personalized drug treatments.

As the vertebrate embryonic axis elongates, there is a fine spatial separation of the temporal events of differentiation that facilitates investigation of distinct steps in neural differentiation [1]. At the tail end of the embryo epiblast, cells adjacent to the rostral primitive streak (caudal lateral epiblast, CLE) can give rise to neural or paraxial mesoderm cell fates, depending on whether they become displaced rostrally to form the neural plate or gastrulate through the primitive streak to make paraxial mesoderm (Figure 1A), while some cells are retained in this region (closest to the primitive streak) and act as stem cell-like axial progenitors $[2,3]$.

A recent paper in Nature from Kondoh and colleagues has begun to shed

Correspondence: Kate G Storey

E-mail: k.g.storey@dundee.ac.uk light on the molecular basis of this neural vs paraxial mesoderm cell fate decision [4]. This elegant study uses a series of mouse mutants to explain the homeotic-like phenotype of mice with a homozygous mutation for the T-box family transcription factor $T b x 6$, which possess two ectopic neural tubes in place of the bilaterally arranged paraxial mesoderm [5]. In doing so, Takemoto et al. reveal that Tbx6 acts in part by downregulating a specific enhancer (N1) of the neural progenitor marker Sox2.

Unlike other Sox1B genes, Sox2 expression extends caudally from the neural tube to include the epiblast cells located adjacent to the primitive streak (Figure 1B). Work from this same group in the chick embryo has shown that the apparently uniform Sox 2 expression along the neural axis is in fact composed of several domains, each regulated by a distinct enhancer and responsive to different signaling pathways [6]. Expression driven by the $\mathrm{N} 1$ enhancer is found around and flanking the primitive streak and is promoted by FGF and Wnt signaling [4] and inhibited by caudal BMP signaling [7]. These signals focus its activity in the so called caudal neural plate and CLE (epiblast just rostral and adjacent to the primitive streak). In contrast, Tbx6 is expressed in the primitive streak and newly emerging paraxial mesoderm.

Analysis of the expression of the N1 enhancer-driven transgene encoding Egfp in wild-type mice showed that enhancer activity is downregulated as cells transit the primitive streak. However, in the Tbx6 mutant, enhancer N1 activation persists in the emerging paraxial mesoderm compartment, while the domains of other Sox1B genes are unchanged. This suggests that Sox 2 $\mathrm{N} 1$ enhancer activity is responsible for failure to switch off the neural programme when Tbx6 is lost. This is tested in mouse mutants homozygous for the N1 region of the Sox2 locus and Tbx6, which now lack ectopic Sox 2 expression in the paraxial mesoderm compartment and these mutant embryos fail to make ectopic neural tubes. Importantly, these mice also fail to express mesodermal genes, even though the neural programme is no longer aberrantly active, indicating that Tbx6 is required both to inhibit N1 activity and to promote mesoderm specification. It will be intriguing to determine what character this non-neural, non-paraxial mesoderm tissue has acquired, as supplementary data indicates it also lacks endodermal markers.

The authors further demonstrate that mis-expression of Sox 2 specifically in the caudal paraxial mesoderm, using a tissue specific enhancer of the gene Delta1, is also sufficient to transform presumptive mesoderm into neural tissue. It would be interesting to know now whether this is mediated by Tbx6 loss? 


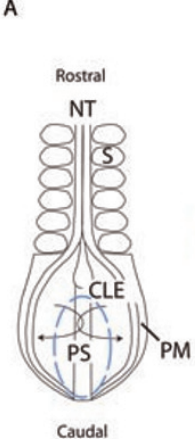

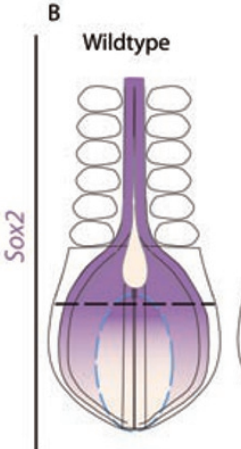

C
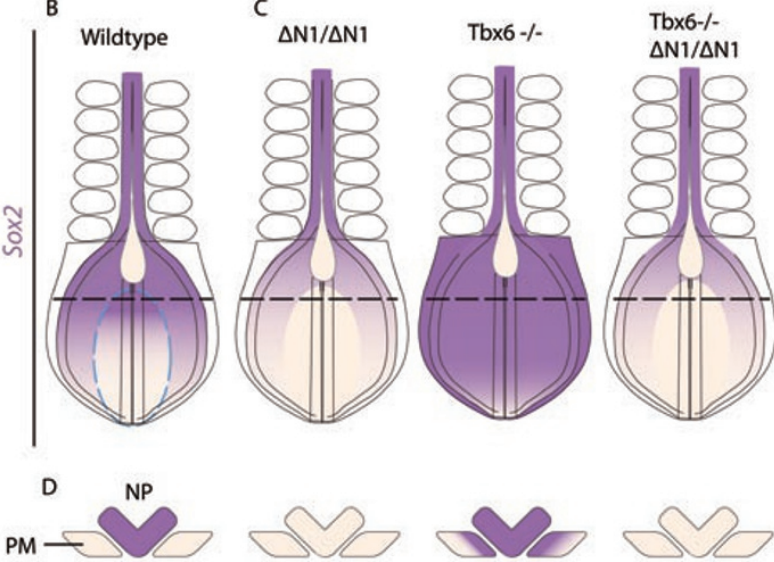
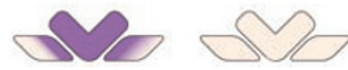

Figure 1 Sox 2 expression is regulated by Tbx6 via Sox2 enhancer N1. Sox2 expression in paraxial mesoderm is supressed by Tbx6 via inhibition of enhancer N1. (A) Schematic diagram of the caudal part of developing mouse embryo. (B) Sox2 is normally expressed in the rostral CLE (dashed blue oval) in wildtype embryos. This expression is driven by enhancer $\mathrm{N} 1$ as deletion of the enhancer abolishes Sox2 expression in the CLE (C). Tbx6-/- mutation leads to ectopic Sox2 expression in the paraxial mesoderm and this is lost in Tbx6-1$\Delta \mathrm{N} 1 / \Delta \mathrm{N} 1$ double homozygous mutant embryos. (D) Illustration of Sox2 expression in transverse sections at indicated levels in (B, C) (dashed lines), predicted from whole mount images in [4]. NT, neural tube; S, somite; CLE, caudal lateral epiblast (dashed blue oval); PS, primitive streak; PM, paraxial mesoderm; NP, neural plate.

The simplest way by which Tbx6 might inhibit $\mathrm{N} 1$ activity is by binding to this Sox2 locus region. However, the authors were unable to establish this and so favour an indirect mechanism of action. A hint of how this might operate comes with the upregulation of Wnt $3 a$ in the presomitic mesoderm of Tbx6 mutants, as Wnt and FGF signaling promote N1 activity [4]. The authors propose a model in which during normal development Tbx6 mediates loss of Sox 2 enhancer N1 activity in cells leaving the primitive streak via repression of Wnt $3 a$ expression.

However, this correlation between increased Wnt signaling and increased neural tissue in the Tbx6 mutant raises some apparent contradictions with respect to other mouse mutants that possess a similar phenotype; ectopic neural tubes in place of paraxial mesoderm. Many of these mutants lack canonical Wnt signaling, see table 1 in [3], correlating low, not high Wnt with a neural over mesodermal cell fate decision. Similarly, mice lacking the retinoic acid catabolising enzyme Cyp26A, which have excess RA $[8,9]$ that represses $\mathrm{Wn}$ $t 3 a[10]$, also form ectopic neural tubes at the expense of mesoderm. In addition, Wnt3a is required for $F g f 8$ expression [11], and mice in which FGFR signaling is blocked also form neural at the expense of mesoderm tissue (although this is associated with a failure to undergo gastrulation). Surprisingly, Kondoh and colleagues further report no significant change in the expression of Fgf4 and Fgf8 transcripts in Tbx6 mutants [4]. Clearly Wnt signaling is important for both neural and mesodermal differentiation and it will be essential in the future to resolve these puzzling findings and determine the link between Tbx6 and Wnt3a transcription. A next step might be to examine readouts of Wnt and FGF signaling and to establish the precise timing and location of any changes in activity levels with respect to the onset of ectopic N1 enhancer activity in Tbx6 mutants.

\section{References}

1 Diez del Corral R, Olivera-Martinez I, Goriely A, Gale E, Maden M, Storey K. Opposing FGF and retinoid pathways control ventral neural pattern, neuronal differentiation, and segmentation during body axis extension. Neuron 2003; 40:65-79.

2 Tzouanacou E, Wegener A, Wymeersch FJ, Wilson V, Nicolas JF. Redefining the progression of lineage segregations during mammalian embryogenesis by clonal analysis. Dev Cell 2009; 17:365376.

3 Wilson V, Olivera-Martinez I, Storey KG. Stem cells, signals and vertebrate body axis extension. Development 2009; 136:1591-1604.

4 Takemoto T, Uchikawa M, Yoshida M, et al. Tbx6-dependent Sox2 regulation determines neural or mesodermal fate in axial stem cells. Nature 2011; 470:394-398.

5 Chapman DL, Papaioannou VE. Three neural tubes in mouse embryos with mutations in the T-box gene Tbx6. $\mathrm{Na}$ ture 1998; 391:695-697.

6 Uchikawa M, Ishida Y, Takemoto T, Kamachi Y, Kondoh H. Functional analysis of chicken Sox2 enhancers highlights an array of diverse regulatory elements that are conserved in mammals. Dev Cell 2003; 4:509-519.

7 Takemoto T, Uchikawa M, Kamachi Y, Kondoh H. Convergence of Wnt and FGF signals in the genesis of posterior neural plate through activation of the Sox 2 enhancer N-1. Development 2006; 133:297-306.

8 Abu-Abed S, Dolle P, Metzger D, Beckett B, Chambon P, Petkovich M. The retinoic acid-metabolizing enzyme, CYP26A1, is essential for normal hindbrain patterning, vertebral identity, and development of posterior structures. Genes Dev 2001; 15:226-240.

9 Sakai Y, Meno C, Fujii H, et al. The retinoic acid-inactivating enzyme CYP26 is essential for establishing an uneven distribution of retinoic acid along the anterio-posterior axis within the mouse embryo. Genes Dev 2001; 15:213-225

10 Shum AS, Poon LL, Tang WW, et al. Retinoic acid induces down-regulation 
of Wnt-3a, apoptosis and diversion of tail bud cells to a neural fate in the mouse embryo. Mech Dev 1999; 84:17-
30.

11 Aulehla A, Wehrle C, Brand-Saberi B, et al. Wnt3a plays a major role in the segmentation clock controlling somitogenesis. Dev Cell 2003; 4:395-406. 